\title{
Vascular and Degenerative Dementia Convergences More Than Differences
}

\author{
Ildefonso Rodríguez Leyva* \\ Faculty of Medicine, Autonomous University of San Luis Potosí, Mexico
}

*Corresponding author: Ildefonso Rodríguez Leyva, Head of the Neurology Service, Central Hospital "Dr. Ignacio Morones Prieto", Faculty of Medicine, Autonomous University of San Luis Potosí, San Luis Potosí, SLP Mexico.

Received Date: January 06, 2020

Published Date: January 14, 2020

\section{Introduction}

Minimal and significant cognitive impairment can have both a vascular and a degenerative origin; both problems must converge in many situations. The brain weighs only $2 \%$ of our body but occupies $20 \%$ of oxygen and body blood flow. The structural alterations that include; Changes in blood supply (macro and microcirculation), in neuronal activity, in molecular changes (proteinopathies), and neurotransmission occur in both situations in both vascular dementia and neurodegenerative. Many of the risk factors for both pathologies coincide, especially age [1].

\section{Convergence}

Autopsies of brains of patients with Alzheimer's frequently have vascular pathology, consisting on degenerative microangiopathy, amyloid angiopathy, cerebral infarctions, and intracerebral hemorrhages.

Vascular disease is present in $80 \%$ of Alzheimer's patients. Furthermore, multiple risk factors converge [2].

Genetic factors, such as APP mutations (chromosome 19). Presenilin 1 (chromosome 14). Presenilin 2 (chromosome 2). APOE (apoE4 allele, chromosome 19). Polymorphisms in secretase and peptidase genes. Notch 3, mutations of Beta synthetase, alphagalactosidase could be fought with prevention measures or have a protective environment: Antihypertensive treatment, use of statins, antioxidants (vitamins C and E). Eating a Mediterranean or at least having a healthy diet, avoiding the consumption of polyunsaturated fats, increasing fish intake, promoting social activity, having a high level of education and physical activity, and a chronic use of non-steroidal anti-inflammatory drugs could prevent neurodegeneration.

\section{Shared Risk Factors}

Many risk factors for dementia are shared: Hypertension, Diabetes Mellitus, obesity in adulthood, smoking, similar diet, sedentary lifestyle, poor education, socioeconomic status, occupation/leisure activities, alcohol/toxins/medicaments, exposure to environmental contamination, sleep disorders (especially deprivation), hearing loss, head trauma, and the social isolation that the older adult often faces. There is also an interaction between genetics and the environment that favors the presence of both problems. Stroke often goes unnoticed if it affected a quiet brain area, and many times it turns out that the patient with Alzheimer's disease had concomitantly cerebral vascular disease in the autopsy. We will analyze some of the risk factors that lead us to present both types of dementia and which are not usually insisted on:

\section{Head trauma}

There is a broad spectrum in the severity of head trauma which can be from mild to severe, and this becomes more evident if there is a structural lesion injury that can be detected by an imaging study (be it CT or MRI), both the presence of micro bleeding and severe traumatic lesions may deteriorate cognition. In cranial trauma, there is usually a functional interruption in the blood-brain barrier that favors inflammation. We well know that repeated cranial trauma favors the presence of tauopathy that is indistinguishable from that presented in Alzheimer's Disease. The subject who has repeated cranial trauma increases the risk of dementia between two to four times compared to the unexposed population [3].

\section{Depression}

There is a directly proportional relationship between the number of episodes of depression and the increased risk of dementia. The percentage of the depressive syndrome is higher in recent years in those who are diagnosed with dementia. It is known that depression increases the production of hormones associated with stress. In addition to decreasing levels of neural growth factors, a decrease in hippocampal volume has been found in the depressed 
population. On the other hand, it is known that antidepressants decrease amyloid production, in addition to increasing BDNF and neurogenesis [4,5].

\section{Alcohol}

It has been known for centuries that dementia is related to alcohol (there are even multiple syndromes that associate alcoholdementia: Korsakoff, Wernicke, Marchiafava-Bignami). In all of them, the leading clinic is undoubtedly the presence of significant cognitive impairment. The structural modifications are found to coincide in all of them although they usually have some distinctive characteristics; generalized atrophy, hippocampus reduction, white matter damage. However, minimum to modest consumption of alcohol offers us minimum to moderate protection. Having a healthy diet, a higher social class, a higher IQ a better education, and a better general level of health, possibly protects the drinker from alcohol being more harmful [6].

\section{Sleep}

Individuals suffering from obstructive sleep apnea (OSA) have twice the frequency of dementia; in them, there is a loss of the circadian rhythm and the sleep-wake relationship; this is associated with progressive cognitive impairment. The presence of REM sleep behavior disorder has been significantly related to Parkinson's disease. It is known that sleeping less than five or more than 9 hours is associated with cognitive impairment. Sleep deprivation increases overnight amyloid-beta production (25-30\%). It is also known that amyloid removal occurs especially during slow-wave sleep. Chronic sleep deprivation increases phosphorylation of the tau protein and increases the presence of biomarkers of amyloid pathology in the CSF, thus facilitating neurodegenerative pathology $[7,8]$.

\section{Loneliness}

Although itis discussed whether it is a risk factor, there is growing evidence that it is associated, it is not known what the reason is but possibly the lack of social interaction or intellectual activity. It is also known that loneliness is associated with an increased risk of hypertension, coronary heart disease and depression. There is an increase in the production of urinary catecholamines, and there is an increase in oxidative stress. Social isolation and loneliness of patients are risk factors for dementia and are potentially modifiable factors for a cognitive malfunction that could be treated $[9,10]$.

\section{Gender}

Women have an epidemiologically speaking increased risk of suffering from Alzheimer's disease. One in six women at age 60 lives with cognitive impairment. African-American women have twice the prevalence of dementia than Caucasian women. The survival of women is higher than that of men, and they represent $62 \%$ of people who are over 80 years old. Population aging is particularly rapid in Africa, Latin America, the Caribbean, and Asia. The prevalence of dementia worldwide is increasing. It is expected that by 2050 , more than two-thirds of people with dementia will live in developing countries. The impact of the problem on women will be more significant than for men [11].

\section{Hearing loss}

Hyperacusis is a risk factor recognized until recently. Hearing loss greater than $25 \mathrm{~dB}$ increases the risk of dementia. The problem is of high prevalence since, in a similar way to presbyopia, the presence of presbyacusis occurs in $32 \%$ of subjects older than 55 years. The risk of cognitive impairment increases with the most severe hearing loss. For each hearing loss of $10 \mathrm{~dB}$, the risk of dementia increases by $20 \%$ [12].

\section{Education}

Stimulate cognition through an environment that favors education protects against cognitive impairment. High schooling against having less than five years of education reduces the risk of dementia by $80 \%$. Having challenging occupations both at work and in recreation may protect against having dementia. Does having a better education decline the risk of dementia? [13,14].

\section{Exercise}

It is known that there is a strong relationship between practicing physical exercise and improving cognitive reserve. Physical exercise improves the ability to acquire knowledge, playing sports decreases the risk of dementia, as long as it does not favor repeated cranial trauma (boxing, American football, soccer), the exercise maintains the structure and not only brain function and has even been proposed to increase the hippocampal volume. In addition to that, it increases the production of neurotrophic factors $[15,16]$.

\section{Conclusion}

Given a scenario where it seems unquestionable that the incidence and prevalence of dementia increases in the general population by increasing its survival, we are forced to implement measures that help us increase the educational level of the population. Creating programs that facilitate the performance of both physical and mental exercise and improving the population's diet (and possibly settling on a Mediterranean-style diet) therefore preventing diseases such as diabetes mellitus and obesity. It is the obligation of all of those who work in Health to promote the prevention of hypertension, diabetes mellitus, overweight, smoking, alcoholism, avoiding sedentary life in ourselves and in those we serve. We must identify atypical risk elements such as insomnia, OSA and REM behavioral disorder. We must treat hearing loss early when it occurs. Also, to be aware of the importance of going to the primary physician in situations that carry a lack of exercise as joint pain. Insist on the patient and his family on the importance of avoiding loneliness. The use of pharmacological measures such as metformin, statins, anticholinesterase drugs, and memantine may be useful, but like everything else in medicine, prevention may be much better than treatment.

\section{Acknowledgement}

None. 


\section{Conflict of Interest}

No conflict of interest.

\section{References}

1. Raj N Kalaria (2010) Vascular basis for brain degeneration: faltering controls and risk factors for dementia, Nutrition Reviews 68(2): S74S87.

2. Toledo JB, Arnold SE, Raible K, Brettschneider J, Xie SX, et al. (2013) Contribution of cerebrovascular disease in autopsy confirmed disease cases in the National Alzheimer's Coordinating Centre. Brain 136(9): 2697-2706.

3. Shively S, Scher AI, Perl DP, Diaz-Arrastia R (2012) Dementia resulting from traumatic brain injury: what is the pathology? Archives of Neurology 69(10): 1245-1251.

4. Fortin JP, Cullen N, Sheline YI, Taylor WD, Aselcioglu I, et al. (2018) Harmonization of cortical thickness measurements across scanners and sites. Neuroimage 167: 104-120.

5. Brier MR, Gordon B, Friedrichsen K, McCarthy J, Stern A, et al. (2016) Tau and $A \beta$ imaging, CSF measures, and cognition in Alzheimer's disease. Science translational medicine 8(338): 338ra66-338ra66.

6. Topiwala A, Allan CL, Valkanova V, Zsoldos E, Filippini N, et al. (2017) Moderate alcohol consumption as a risk factor for adverse brain outcomes and cognitive decline: a longitudinal cohort study. BMJ 357: j2353.

7. Chee MW1, Chuah LY (2008) Functional neuroimaging insights into how sleep and sleep deprivation affect memory and cognition. Current opinion in neurology 21(4): 417-423.
8. Sprecher KE, Koscik RL, Carlsson CM, Zetterberg H, Blennow K, et al. (2017) Poor sleep is associated with CSF biomarkers of amyloid pathology in cognitively normal adults. Neurology 89(5): 445-453.

9. Holwerda TJ, Deeg DJ, Beekman AT, van Tilburg TG, Stek ML, et al. (2014) Feelings of loneliness, but not social isolation, predict dementia onset: results from the Amsterdam Study of the Elderly (AMSTEL). J Neurol Neurosurg Psychiatry 85(2): 135-142.

10. John T. Cacioppo, Stephanie Cacioppo (2014) Social relationships and health: The toxic effects of perceived social isolation. Social and personality psychology compass 8(2): 58-72.

11. Erol Rosie, Dawn Brooker, Elizabeth Peel (2015) Women and dementia: A global research review.

12. Rhett $\mathrm{S}$ Thomson, Priscilla Auduong, Alexander T Miller, Richard K Gurgel (2017) Hearing loss as a risk factor for dementia: a systematic review. Laryngoscope investigative otolaryngology 2(2): 69-79.

13. Katzman Robert (1993) Education and the prevalence of dementia and Alzheimer's disease. Neurology 43(1): 13-20.

14. Stern Y, Gurland B, Tatemichi TK, Tang MX, Wilder D, et al. (1994) Influence of education and occupation on the incidence of Alzheimer's disease. Jama 271(13): 1004-1010.

15. Petrovitch Helen, Lon White (2005) Exercise and cognitive function. The Lancet Neurology 4(11): 690-691.

16. David W Dunstan, Michael J.Wheeler, Kathryn A Ellis, Ester Cerince, Daniel J Green (2018) Interacting effects of exercise with breaks in sitting time on cognitive and metabolic function in older adults: Rationale and design of a randomized crossover trial. Mental Health and Physical Activity 15: 11-16. 